\title{
Predictors of involuntary patients' satisfaction with care: prospective study $\dagger$
}

Emma Bainbridge, Brian Hallahan, David McGuinness, Patricia Gunning, John Newell, Agnes Higgins, Kathy Murphy and Colm McDonald

\section{Background}

Involuntary admission can be traumatic and is associated with negative attitudes that persist after the episode of illness has abated.

\begin{abstract}
Aims
We aimed to prospectively assess satisfaction with care at the points of involuntary admission and symptomatic recovery, and identify their sociodemographic, clinical and service experience predictors.
\end{abstract}

\section{Method}

Levels of satisfaction with care, and clinical and sociodemographic variables were obtained from a representative cohort of 263 patients at the point of involuntary admission and from 155 of these patients 3 months after termination of the involuntary admission. Data were analysed with multiple linear regression modelling.

\section{Results}

Higher baseline awareness of illness $(B=0.19, P<0.001)$ and older age $(B=0.05, P=0.001)$ were associated with more satisfaction with care at baseline and follow-up. Transition to greater satisfaction with care was associated with improvements in awareness of illness $(B=0.13, P<0.001)$ and in symptoms $(B=$ $0.05, P=0.02)$, as well as older age $(B=0.04, P=0.01)$. Objective coercive experiences were not associated with variation in satisfaction with care.

\section{Conclusions}

There is wide variation in satisfaction with coercive care. Greater satisfaction with care is positively associated with clinical variables such as increased awareness of illness.

\section{Declaration of interest}

None.

\section{Keywords}

Mental Health Act; psychosis; satisfaction; insight; involuntary admission.

\section{Copyright and usage}

(c) The Royal College of Psychiatrists 2018. This is an Open Access article, distributed under the terms of the Creative Commons Attribution-NonCommercial-NoDerivatives licence (http://creativecommons.org/licenses/by-nc-nd/4.0/), which permits noncommercial re-use, distribution, and reproduction in any medium, provided the original work is unaltered and is properly cited. The written permission of Cambridge University Press must be obtained for commercial re-use or in order to create a derivative work.
Involuntary admission to a psychiatric in-patient unit is one of the most ethically challenging practices in medicine and is associated with fear, exclusion, powerlessness and low satisfaction with care. ${ }^{1-5}$ Follow-up studies indicate a wide variation in the extent to which individuals regard their involuntary admission as justified and/or treatment as beneficial, with a tendency for satisfaction with care rates to increase with time since the admission, ${ }^{6,7}$ although the opposite has also been reported. ${ }^{8}$ The determinants of higher satisfaction with care among involuntarily admitted patients also remain uncertain because of a lack of consistent findings from studies to date. Studies have reported associations between perceived justified involuntary admission and higher satisfaction levels with symptomatic improvement, ${ }^{9}$ male gender, diagnosis other than schizophrenia $^{7}$ and better awareness of illness. ${ }^{1,10}$ Investigations as to whether the objective use of physical force is associated with lower satisfaction with care among patients has produced conflicting evidence..$^{9-12}$ Other potentially service-related factors that have been linked to better patient satisfaction with care include a positive therapeutic relationship with staff $^{5}$ and involvement of patients in clinical decision-making. ${ }^{4,13}$ Rates of involuntary admission vary considerably across Europe along with differing legislative and mental health systems. ${ }^{14}$ Legislation in the Republic of Ireland was modernised in 2006 (Mental Health Act 2001) and incorporated several protections for patients, including the right to automatic independent review of admission by a mental health review tribunal and closer scrutiny of coercive practices. However, it is

$\dagger$ See this issue unknown whether the improved alignment of legislation with current human rights principles in Ireland has had any effect on patient attitudes toward the justification for their involuntary admission or the quality of care received during it. The aims of this study were to (a) assess satisfaction with care among a representative sample of involuntary patients at admission, and when their symptoms had improved; and (b) identify what predicts satisfaction with care and change in satisfaction with care over time.

\section{Method}

\section{Study design}

The study was a prospective observational study conducted between 2011 and 2014. Patients were assessed initially upon involuntary admission as soon as they were able to participate in the assessments (baseline), and again approximately 3 months following termination of their involuntary admission order (follow-up). The follow-up assessment time point was chosen to balance the individual's likely ability to freshly recollect the episode of care and symptomatic improvement after an episode of illness exacerbation (because many patients continue as voluntary in-patients or with intensive community treatment for a period after termination of their involuntary admission). There was flexibility in the timing of reassessments, which were delayed until after discharge if the patient was still an in-patient or had been readmitted at the time of attempted re-recruitment. 


\section{Participants}

The study was carried out across three psychiatric in-patient units in the west of Ireland, covering a population of 314000 individuals and representative of the differing types of in-patient services that characterise in-patient psychiatric care: the first attached to a tertiary referral academic hospital, the second attached to a regional hospital and the third a standalone psychiatric facility. Recruitment was through direct referral by clinicians or self-referral in response to study brochures distributed by ward staff. Attempts were made to recruit all eligible involuntarily admitted patients over the study period, subject to the following exclusion criteria: aged under 18 years or inability to engage in the interview process because of severity of mental disorder or cognitive impairment. Individuals were eligible to participate each time they experienced an involuntary admission during the study period.

Ethical approval was obtained before the commencement of this study from the Clinical Research Ethics Committee for Galway University Hospitals (ref CA524) and the National University of Ireland, Galway (ref 11/MAR/04). All participants provided written informed consent. Verbal assent was obtained from those unable to give informed consent at the initial interview phase if they were considered by the interviewer to lack capacity on the basis of their mental state. Informed consent was sought from these participants at a later stage when the individual had improved symptomatically. Those who assented but did not later provide informed consent were excluded from the study $(n=3)$.

\section{Procedures and measures}

Sociodemographic, clinical and service experience data were collected at each time point. A number of validated psychometric assessments of attitudes toward care and clinical characteristics were undertaken. These included assessments of symptoms (Brief Psychiatric Rating Scale, clinician-rated), ${ }^{15}$ functioning (Global Assessment of Functioning (GAF), clinician-rated), ${ }^{16}$ awareness of illness (Schedule for the Assessment of Insight in Psychosis (SAI-E), clinician-rated), ${ }^{17}$ attitudes toward medical treatment (Hogan Drug Attitude Scale, self-report) ${ }^{18}$ and satisfaction with care (Client Assessment of Treatment Scale (CAT), self-report). ${ }^{19}$ To further characterise attitudes toward the experience of involuntary admission, perceptions of care and treatment were assessed by the MacArthur Admission Experience Survey (self-report), ${ }^{20}$ which measures levels of perceived coercion, perceived pressure and procedural justice, and the Coercion Ladder (self-report), ${ }^{21}$ which assesses beliefs regarding levels of coercive treatment.

\section{Follow-up assessments}

All baseline assessments were repeated at the follow-up. In addition, formal diagnosis was established by the Structured Clinical Interview for DSM-IV Disorders, ${ }^{22}$ and quality of life was assessed by the Heinrichs Quality of Life Scale (clinician-rated). ${ }^{23}$ Additional service experience data during the admission was collected at follow-up, including attendance at mental health review tribunals and use of coercive measures such as restraint, seclusion or forced intramuscular injections. The clinician-rated quantitative assessments were carried out by a researcher (E.B.) who was independent of the individuals' clinical care and completed clinical assessments blind to the participant-rated scores on their attitudes toward care and coercive practices.

\section{Statistical analyses}

Initially, all data were cleaned and checked for outliers by generating numerical, tabular and graphical summaries at baseline and followup. Case profile plots were used to identify any overly influential changes in each variable over time. Clinical measures across time points for individuals who were successfully re-recruited were analysed by paired $t$-tests. The significance level was set at $P=0.05$ for these statistical analyses.

The primary response of interest was satisfaction with care scores (as assessed by the mean CAT score). Because this behaved as a continuous variable, multiple regression models were used to explore the relationship between this variable and potential predictor variables. Given that satisfaction levels were expected to alter between baseline and follow-up, three models were explored to assess the effect of explanatory variables with satisfaction over time: (a) predictor variables associated with satisfaction with care at baseline, (b) predictor variables associated with satisfaction with care at follow-up and (c) predictor variables associated with change in satisfaction with care between baseline and follow-up.

The variables chosen were those that we expected might reasonably explain a proportion of the variance in satisfaction levels in the light of the existing literature, and incorporated a range of commonly acquired clinical, sociodemographic and service-related data. All of the included variables were considered potential explanatory variables rather than confounders. At baseline, the variables included were symptoms (Brief Psychiatric Rating Scaling), awareness of illness (SAI-E), functioning (GAF), diagnosis, age, gender, whether an admission order was completed, the total number of previous involuntary admissions, history of alcohol misuse and history of illicit substance misuse. The variables chosen for the follow-up and changes over time analyses included the above variables and quality-of-life scores (in place of GAF), whether or not the person was subjected to coercive measures (restraint and/or seclusion and/or forced intramuscular medication), the length of time the person was detained for and whether their involuntary admission had been reviewed by a mental health review tribunal. The perceptions of care variables, as assessed by the MacArthur Admission Experience Survey, were not included in the multivariable analysis because they were considered outcome variables (similar to satisfaction with care scores) rather than potential explanatory variables related to the illness, clinical features or services.

When modelling satisfaction with care at follow-up, the regression model included the primary response at baseline as a covariate in addition to the pre-selected predictor variables. To investigate how changes in satisfaction with care over time were associated with changes in the predictor variables, the change score in the response was regressed on the change score in the set of predictor variables, where relevant.

The likelihood ratio test and Akaike information criterion were used to identify the correct functional form for each explanatory by fitting higher-order splines for each covariate. The assumptions underlying each regression model were assessed with suitable residual plots. As an addition to the regression models, regression trees using conditional inference ${ }^{24}$ were used to identify subgroups in the sample that explained significant components of variability in the change in satisfaction variable. Statistical analyses were completed with R-package (version 3 for Windows). ${ }^{25} \mathrm{~A}$ statistical significance threshold of $P<0.05$ was chosen for these multivariable analyses, whereas goodness of fit was assessed by adjusted $R^{2}$ score.

\section{Results}

Of 460 individual presentations over the study period, 69 were excluded ( 30 had cognitive impairment, 17 were transferred out of area or absconded before assessment and 22 were too mentally unwell or unable to participate) and 128 refused to participate. In total, 263 individuals (67\% of eligible participants) completed baseline assessments, on average within 9 days of admission, and 155 
individuals completed follow-up assessments. Sociodemographic and clinical data are presented in Table 1. The mean age of participants at baseline assessment was 43 years (s.d. 15.6, range 19-88). A total of $35(13 \%)$ individuals had more than one involuntary admission during the study period. For those transferred from the community on an admission order, the majority of applications for involuntary admission were made by a relative ( $n=110,52 \%)$. Other applicants were police $(n=85,39 \%)$, authorised officers $(n=4,2 \%)$ and other persons $(n=16,8 \%)$. An assisted admissions team (consisting of psychiatric nurses with or without police support) was involved in transfer to hospital for $86(40 \%)$ participants admitted from the community. In 58 participants (22\%), the application for an involuntary admission was not supported by a consultant psychiatrist within the $24 \mathrm{~h}$ assessment period under the legislation, and the involuntary admission was discontinued. At initial assessment, 232 (88\%) participants provided informed consent and 31 (12\%) participants provided assent.

\section{Clinical characteristics}

On formal diagnostic assessment at follow-up, 129 (83\%) participants fulfilled the criteria for a psychotic illness. During the involuntary admission process, 69 (26.2\%) participants were subjected to one or more objective coercive measures of physical restraint $(n=56)$, seclusion $(n=33)$ or forced intramuscular injection of medication $(n=48)$. Participants demonstrated statistically significant substantial improvements in clinical symptoms, awareness of illness, functioning and attitudes toward prescribed medication treatment between baseline assessment and follow-up (Table 2). There was no significant difference in baseline values of symptoms $(t=-1.79, P=0.07)$, functioning $(t=0.37, P=0.71)$ or awareness of illness $(t=-0.83, P=0.41)$ between the 155 individuals who participated in follow-up and the 108 individuals who did not. Participants were asked at each time point if they believed that their involuntary admission had been necessary; $42.2 \%$ agreed with this statement at initial interview and $65.3 \%$ agreed at follow-up.

\section{Satisfaction with involuntary care}

Satisfaction with care was highly variable, with overall satisfaction levels tending toward positive and a mean satisfaction with care score of $>6$ out of 10 at both time points, but it also demonstrated statistically significant improvement between baseline and followup (Table 2). Participants who left out subscale items tended to be more negative in their responses to other items; for example, 76 individuals elected not to answer the subscale relating to 'believe their care is right' at baseline and their mean satisfaction with care score was 5.28 (s.d. 3.03), with a score on the subscale 'receiving right treatment' of 3.17 (s.d. $3.57, n=48$ ). Significant improvements over time on the subscales comprising the satisfaction with care score were found for satisfaction with receiving the right treatment, engagement with the treating psychiatrist, believing that right medication was administered and treatment being helpful.

Significant reductions in perceived pressure and procedural justice were reported by participants between baseline and followup assessments (Table 2). Those who considered their admission necessary at follow-up had significantly higher mean satisfaction with care scores than those who did not (mean 7.82 (s.d. 2.01) $v$. 4.45 (s.d. 3.06), $t=-8.06, P<0.001$ ). Satisfaction with care scores were strongly negatively correlated with procedural justice scores at baseline $(r=-0.53, P<0.001)$ and follow-up $(r=-0.69, P<0.001)$, and were more weakly correlated with perceived coercion and perceived pressure (baseline: $r=-0.31, P<0.001$ and $r=-0.21$, $P=0.001$, respectively; follow-up: $r=-0.16, P=0.06$ and $r=-0.18$, $P=0.03$, respectively). There was no significant difference in mean satisfaction with care scores at follow-up between those who had been subject to objective coercive practices and those who had not (mean 6.38 (s.d. 2.90) v. 6.81 (s.d. 2.89), $t=0.89, P=0.38$ ).

\section{Multivariable analysis}

Baseline predictors of satisfaction with care during the involuntary admission

Multivariable analysis of participants' data at baseline demonstrated that more satisfaction with care was significantly associated with greater awareness of illness, older age and having a history of harmful alcohol use after adjusting for several other variables, including level of symptoms, gender, diagnosis, functioning, status of admission, number of previous involuntary admissions and history of illicit substance use (Table 3 and Fig. 1(a)). The variance explained by this model was $27 \%$.

\section{Baseline predictors of satisfaction with care toward involuntary admission assessed at follow-up}

Greater satisfaction with involuntary treatment and care when reassessed at follow-up was significantly associated with greater satisfaction with care at baseline, and with greater awareness of illness at baseline, having more symptoms at baseline, older age and having fewer previous involuntary admissions after adjusting for several other potentially confounding variables (Table 3 ). The variance explained by this model was $34 \%$. Notably, there was no significant association between satisfaction with involuntary care and the actual use of objective coercive measures during the admission (forced injection, seclusion or physical restraint), the duration of the involuntary admission or whether the individual was provided with an independent review by a mental health tribunal. Given the strong association between satisfaction with care at baseline and at follow-up, the analysis was repeated excluding this variable to assess what clinical variables during admission were predictive of satisfaction with care at follow-up. This model explained 25\% of the variance and the significant predictor variables of satisfaction with care remained greater awareness of illness $(B=0.21$, s.e. 0.06 , $P<0.001)$, higher level of symptoms at baseline $(B=0.06$, s.e. $0.02, P=0.009)$, older age $(B=0.07$, s.e. $0.02, P=0.001)$, a history of alcohol misuse $(B=1.35$, s.e. $0.55, P=0.02)$ and fewer involuntary admissions $(B=-0.11$, s.e. $0.06, P=0.05)$.

\section{Predictors of improvement in satisfaction with care over time}

We found that lower satisfaction with care at baseline, greater improvements in awareness of illness over time, greater improvement in symptoms over time, older age and having a diagnosis of a nonpsychotic illness were associated with a change to higher levels of satisfaction with care over time, after adjusting for multiple other variables (Table 3 and Fig. 1(b)). The variance explained by this model was $53 \%$. There was no significant association between change in satisfaction levels toward involuntary care over time and the use of objective coercive measures during the admission, the duration of the involuntary admission, quality-of-life scores or whether the individual was provided with an independent review by a mental health tribunal. Figure 2 displays a regression tree that incorporates cut-off values of the continuous variables that were optimal in dividing participants into groups on the basis of their change in satisfaction with care over time. In this analysis, gender also had a modulating effect on the association, in that women with less improvement in symptoms and awareness of illness displayed a worsening of satisfaction with care scores over time. 


\begin{tabular}{|c|c|c|}
\hline Demographic variable & $\begin{array}{c}\text { Baseline }(n=263) \\
n(\%)\end{array}$ & $\begin{array}{c}\text { Follow-up }(n=155) \\
n(\%)\end{array}$ \\
\hline Male gender & $167(63.5)$ & $101(65.2)$ \\
\hline \multicolumn{3}{|l|}{ Age group } \\
\hline 18-24 years & $29(11.0)$ & $18(11.6)$ \\
\hline $25-44$ years & $123(46.8)$ & $74(47.7)$ \\
\hline $45-64$ years & $86(32.7)$ & $52(33.5)$ \\
\hline$\geq 65$ years & $25(9.5)$ & $11(7.1)$ \\
\hline \multicolumn{3}{|l|}{ Marital status } \\
\hline Single & $161(61.2)$ & $96(61.9)$ \\
\hline Married & 40 (15.2) & $23(14.8)$ \\
\hline Significant relationship & $15(5.7)$ & $3(1.9)$ \\
\hline Divorced/separated & $36(13.7)$ & $23(14.8)$ \\
\hline Widowed & $11(4.2)$ & $10(6.5)$ \\
\hline Living alone & $94(35.7)$ & $54(34.8)$ \\
\hline English as first language & $241(91.6)$ & $141(91.0)$ \\
\hline \multicolumn{3}{|l|}{ Educational level attained } \\
\hline Completed primary level & $110(41.8)$ & $70(45.2)$ \\
\hline Completed secondary level & $70(26.6)$ & $35(22.6)$ \\
\hline Completed third level & $63(24.0)$ & $33(21.3)$ \\
\hline Completed postgraduate level & $20(7.6)$ & $17(11.0)$ \\
\hline \multicolumn{3}{|l|}{ Status of involuntary admission } \\
\hline Involuntary admission from community & $213(81.0)$ & $106(68.0)$ \\
\hline Transition from voluntary to involuntary in-patient status & $50(19.0)$ & $49(32.0)$ \\
\hline Previous psychiatric history & 225 (85.6) & 132 (85.2) \\
\hline Index admission was first involuntary admission & $119(45.2)$ & $73(47.1)$ \\
\hline \multicolumn{3}{|l|}{ Family history of psychiatric illness } \\
\hline Yes & $122(46.4)$ & $73(47.1)$ \\
\hline No & $80(30.4)$ & $50(32.3)$ \\
\hline Unknown & $61(23.2)$ & $32(20.6)$ \\
\hline History of alcohol misuse & $115(43.7)$ & $64(41.3)$ \\
\hline History of illicit substance misuse & 79 (30.9) & $44(28.4)$ \\
\hline \multicolumn{3}{|l|}{ Type of illicit substance used } \\
\hline Cannabis & $36(45.5)$ & $24(54.5)$ \\
\hline Other & $7(9.0)$ & $5(11.4)$ \\
\hline Cannabis and other substance(s) & $36(45.5)$ & $15(34.1)$ \\
\hline \multicolumn{3}{|l|}{ Primary DSM-IV diagnosis } \\
\hline Schizophrenia & & $47(30.3)$ \\
\hline Bipolar disorder & & $46(29.7)$ \\
\hline Schizoaffective disorder & & $16(10.3)$ \\
\hline Major depressive disorder (three with psychosis) & & $8(5.1)$ \\
\hline Alcohol dependence syndrome & & $8(5.1)$ \\
\hline Borderline personality disorder & & $6(3.9)$ \\
\hline Other psychotic disorder & & $17(11.0)$ \\
\hline No diagnosis/other & & $7(4.5)$ \\
\hline & Mean (s.d.), range & Mean (s.d.), range \\
\hline Age at first admission, years & $32.1(14.7), 13-85$ & $31.8(14.1), 13-85$ \\
\hline \multicolumn{3}{|l|}{ Time spent in hospital for index admission, days } \\
\hline Period of involuntary admission & $25.1(35.9), 1-219$ & $25.3(33.8), 1-181$ \\
\hline Period of total admission & $38.4(39.5), 1-241$ & $39.7(39.2), 1-220$ \\
\hline Time between admission and baseline assessment, days & $8.9(7.7), 1-43$ & $8.3(6.9), 1-34$ \\
\hline Time between termination of involuntary order and follow-up assessment, days & & $93.5(38.5), 6-236$ \\
\hline
\end{tabular}

\section{Discussion}

This study demonstrated that, on average, levels of satisfaction with involuntary care and treatment were in the positive half of the range ( $>6$ on a scale ranging from $0-10$ ), even during baseline assessment shortly after admission, with an overall further small improvement in satisfaction with care when participants were reassessed 3 months after termination of their involuntary admission order. These levels are consistent with some prior studies that used similar instruments. ${ }^{6,79}$ The study also demonstrated that the strongest predictors of higher satisfaction with care, and improvement in such satisfaction levels over time, tended to be the clinical and demographic variables of awareness of illness and older age, and not the service-related variables of being subject to physically coercive force or having the admission independently reviewed by a tribunal.
Despite the relatively high mean scores on the satisfaction with care scale, it was notable that there was a large variance in the scale measures, indicating wide heterogeneity among respondents. A substantial minority of participants displayed persistently poor levels of satisfaction toward their care, even at the 3-month follow-up assessment, which was timed for when they had returned to their usual level of symptoms and functioning after the episode of illness. It is possible that low satisfaction with care is related to general dissatisfaction among patients with mental illness about their treatment and the perceived emphasis on medication and lack of information, ${ }^{26}$ and is not specifically related to the experience of involuntary admission.

Although a minority of individuals agreed that their admission was necessary on admission, this increased substantially to twothirds of the sample who retrospectively viewed their involuntary admission as necessary at follow-up. This is a higher proportion than that reported by Priebe et al (40\%) in a 1-year follow-up of 
Table 2 Structured assessments of clinical and attitudinal variables on participants at baseline and follow-up

Scale

Baseline: mean (s.d.); range ( $n$ )

Symptoms (BPRS total score)

45.62 (12.85); 0-55 ( $n=263)$

Functioning (GAF)

Awareness of illness (SAI-E total score)

$26.49(12.60) ; 8-88(n=263)$

$5.18(4.76) ; 0-22(n=197)$

-0.05 (16.65); -30 to +30

( $n=233)$

Satisfaction with care during involuntary admission (CAT mean score) $6.21(3.16) ; 0-10(n=260)$

(a) Receiving right treatment

(b) Engagement with psychiatrist

(c) Relation with staff

(d) Receiving right medication

(e) Believe care is right

(f) Respected/well regarded

(g) Treatment been helpful

5.87 (3.95); $0-10(n=247)$

5.79 (3.96): $0-10(n=244)$

$7.70(2.94) ; 0-10(n=254)$

$5.10(4.08) ; 0-10(n=222)$

$6.19(3.86) ; 0-10(n=187)$

$7.16(3.41) ; 0-10(n=249)$

5.87 (4.00); 0-10 $(n=260)$

Coercion Ladder

Perceived coercion (MacArthur Admission Interview)

Perceived pressure (MacArthur Admission Interview)

$7.89(2.97) ; 1-10(n=252)$

$4.23(1.39) ; 0-5(n=257)$

$1.35(0.96) ; 0-4(n=257)$

Procedural justice (MacArthur Admission Interview)

9.76 (4.27): $1-16(n=253)$

\begin{tabular}{|c|c|c|}
\hline $\begin{array}{l}\text { Follow-up: mean (s.d.); } \\
\text { range (n) }\end{array}$ & $\begin{array}{l}\text { Paired } \\
t \text {-test }^{\text {a }}\end{array}$ & $P$-value \\
\hline 28.50 (4.48); 0-55 (n=155) & 18.78 & $<0.001$ \\
\hline 67.30 (11.61); 28-90 (n=155) & -32.47 & $<0.001$ \\
\hline 11.27 (7.57); 0-26 ( $n=124)$ & -9.10 & $<0.001$ \\
\hline $5.30(16.52) ;-30$ to $+30(n=145)$ & -5.37 & $<0.001$ \\
\hline $6.68(2.88) ; 0-10(n=152)$ & -2.79 & 0.006 \\
\hline $6.67(3.37) ;(n=137)$ & -2.99 & 0.003 \\
\hline 6.55 (3.49); $(n=140)$ & -3.47 & 0.001 \\
\hline $7.60(2.99) ;(n=146)$ & -0.15 & 0.88 \\
\hline $5.90(3.52) ;(n=123)$ & -3.39 & 0.001 \\
\hline $7.13(3.10) ;(n=95)$ & -1.52 & 0.13 \\
\hline 7.23 (3.23); $(n=143)$ & -0.89 & 0.38 \\
\hline $6.53(3.55) ;(n=138)$ & -2.15 & 0.03 \\
\hline $8.11(2.56) ; 1-10(n=145)$ & -0.044 & 0.965 \\
\hline $4.52(1.15) ; 0-5(n=152)$ & -1.74 & 0.085 \\
\hline $1.01(0.84) ; 0-4(n=152)$ & 4.83 & $<0.001$ \\
\hline $43(3,91) \cdot 2-16(n=150)$ & 4.19 & $<0.00$ \\
\hline
\end{tabular}

BPRS, Brief Psychiatric Rating Scale; CAT, Client Assessment of Treatment (seven subscales; GAF, Global Assessment of Functioning; HDAl, Hogan Drug Attitude Inventory; SAI-E, Scheduled

for Assessment of Insight in Psychosis.

Statistically significant comparisons are highlighted in bold.

a. Statistical comparison performed on those who participated at both time points.

\begin{tabular}{|c|c|c|c|c|c|c|}
\hline \multirow[b]{2}{*}{ Variable } & \multicolumn{2}{|c|}{$\begin{array}{c}\text { Baseline predictors of } \\
\text { baseline satisfaction with } \\
\text { care }\end{array}$} & \multicolumn{2}{|c|}{$\begin{array}{l}\text { Baseline predictors of } \\
\text { follow-up satisfaction } \\
\text { with care }\end{array}$} & \multicolumn{2}{|c|}{$\begin{array}{l}\text { Predictors of change in } \\
\text { satisfaction with care over } \\
\text { time }\end{array}$} \\
\hline & $B$ (s.e.) & $P$-value & $B$ (s.e.) & $P$-value & $B$ (s.e.) & $P$-value \\
\hline Satisfaction with care at baseline (CAT) & - & - & $0.35(0.09)$ & $<0.001$ & $-0.59(0.07)$ & $<0.001$ \\
\hline Awareness of illness (SAI-E) & $0.19(0.05)$ & $<0.001$ & $0.13(0.06)$ & 0.024 & $0.13(0.03)$ & $<0.001$ \\
\hline Symptoms (BPRS) & $-0.04(0.02)$ & 0.06 & $0.08(0.02)$ & $<0.001$ & $0.05(0.02)$ & 0.02 \\
\hline Quality of life (QoL) & - & - & $-0.01(0.01)$ & 0.61 & $-0.01(0.01)$ & 0.21 \\
\hline Functioning (GAF) & $0.02(0.03)$ & 0.35 & & & - & - \\
\hline Age & $0.05(0.02)$ & 0.001 & $0.05(0.02)$ & 0.004 & $0.04(0.02)$ & 0.01 \\
\hline Gender & $0.17(0.44)$ & 0.69 & $-0.95(0.49)$ & 0.06 & $-0.55(0.44)$ & 0.21 \\
\hline Diagnosis affective versus non-affective & $0.22(0.43)$ & 0.61 & $0.30(0.49)$ & 0.53 & $0.71(0.47)$ & 0.13 \\
\hline Diagnosis affective versus other & $0.88(0.73)$ & 0.23 & $1.89(1.18)$ & 0.11 & $2.48(1.08)$ & 0.02 \\
\hline Alcohol misuse & $0.92(0.46)$ & 0.05 & $0.86(0.53)$ & 0.11 & $0.74(0.49)$ & 0.14 \\
\hline Illicit substance misuse & $-0.95(0.56)$ & 0.09 & $-0.33(0.65)$ & 0.61 & $-0.06(0.59)$ & 0.92 \\
\hline Admission status (Admission order completed or not) & $0.21(0.60)$ & 0.73 & $-0.51(0.73)$ & 0.49 & $-0.97(0.67)$ & 0.15 \\
\hline Number of previous involuntary admissions & $-0.07(0.05)$ & 0.15 & $-0.11(0.05)$ & 0.04 & $-0.09(0.05)$ & 0.07 \\
\hline Use of coercive measures & - & - & $0.65(0.54)$ & 0.23 & $0.29(0.50)$ & 0.56 \\
\hline Length of involuntary admission & - & - & $-0.01(0.01)$ & 0.96 & $0.01(0.01)$ & 0.44 \\
\hline Experience of tribunal during index admission & - & - & $-0.35(0.52)$ & 0.50 & $-0.82(0.49)$ & 0.09 \\
\hline \multicolumn{7}{|c|}{$\begin{array}{l}\text { Coercive measures included restraint and/or seclusion and/or forced intramuscular medication. Diagnosis was coded as non-affective, affective or other, and represented by two dummy } \\
\text { variables. } \\
\text { B refers to unstandardized regression coefficient. } \\
\text { BPRS, Brief Psychiatric Rating Scale; CAT, Client Assessment of Treatment; GAF, Global Assessment of Functioning; QoL, Quality of Life Scale; SAI-E, Scheduled for Assessment of Insight in } \\
\text { Psychosis. } \\
\text { Statistically significant comparisons are highlighted in bold. }\end{array}$} \\
\hline
\end{tabular}

involuntarily admitted patients in England, ${ }^{27}$ but within the range reported in a review of 18 follow-up studies that included both prospective and retrospective designs. ${ }^{6}$ Individuals who believed that their involuntary admission was justified, rated either dichotomously or by lower scores on the procedural justice scale, had significantly greater satisfaction with care at both time points. Significant, albeit weaker, associations were found between lower perceived coercion or perceived pressure, and greater satisfaction with care. Similarly, Katsakou et al ${ }^{9}$ found that higher levels of perceived coercion were associated with more dissatisfaction with treatment, and Strauss et $a l^{28}$ reported a significant association between higher levels of perceived coercion and being less satisfied with in-patient treatment during involuntary admission.

In this study, awareness of illness (as assessed by the SAI-E scale) emerged as one of the strongest predictors of greater satisfaction with care at both time points. Additionally, greater improvement in awareness of illness over time was found to be associated with higher levels of satisfaction with the experience of involuntary care at follow-up. These results are consistent with findings of O'Donoghue et $a l^{8,29}$ who reported an association between insight and whether individuals viewed their involuntary admission as necessary. A study by Smith et $a l,{ }^{5}$ which included voluntarily admitted patients, reported that awareness of illness as assessed via self-report was associated with more positive attitudes toward involuntary admission and treatment. Similarly, a small study by Naber et $a l^{1}$ noted that good insight was associated with whether or not individuals viewed their admission as necessary. The unawareness of illness scale used in our study is not a simple binary construct, but consists of several continua, incorporating dimensions of recognition of illness, need for treatment and 


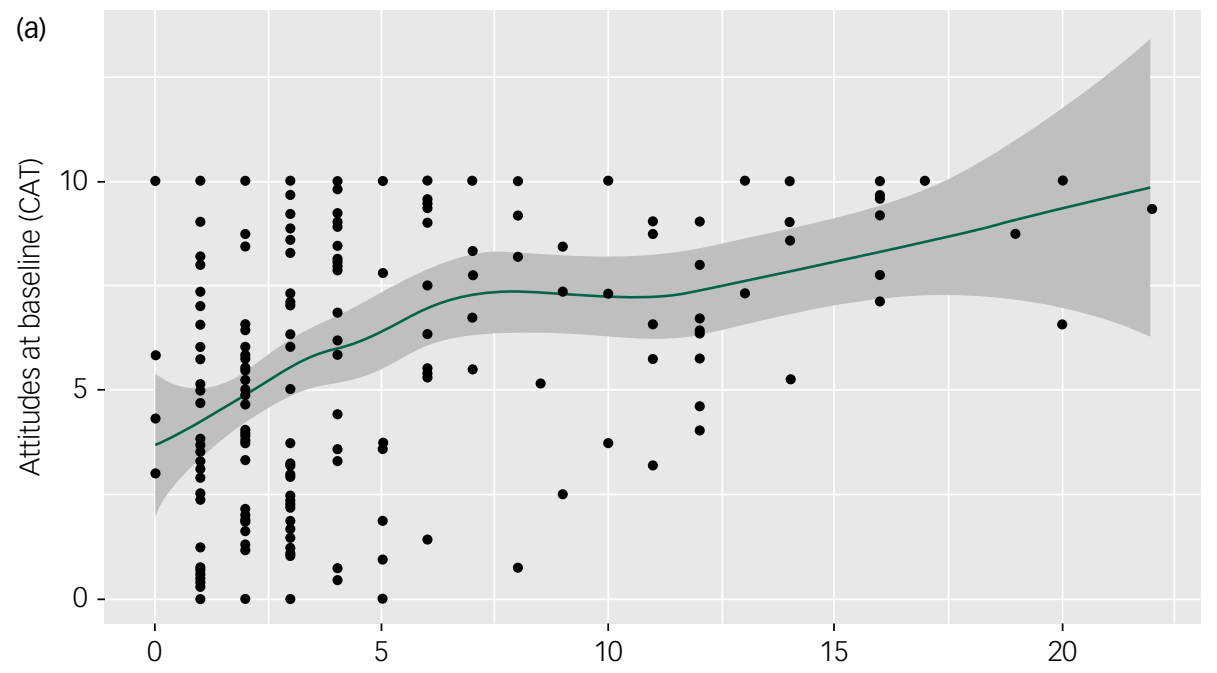

Awareness of illness at baseline (insight)

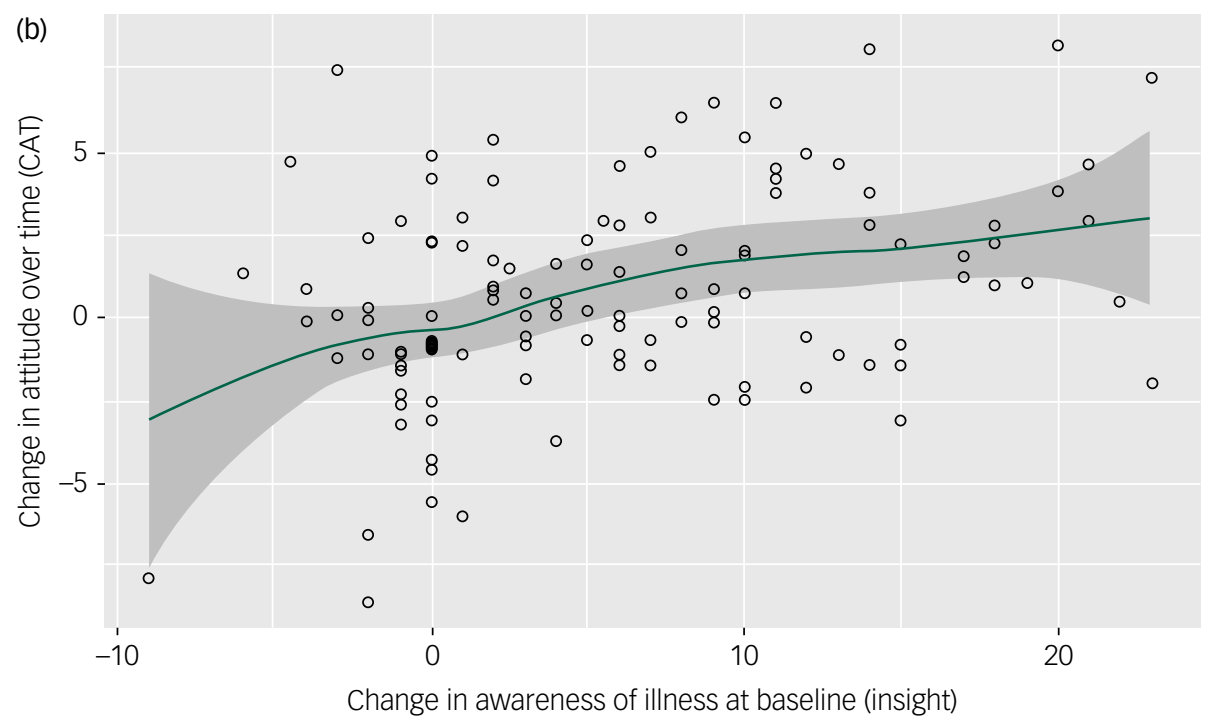

Fig. 1 Association between (a) satisfaction with admission and care at baseline (mean CAT score) and measures of awareness of illness (total Schedule for the Assessment of Insight in Psychosis score) at baseline and (b) change in satisfaction with care over time and change in awareness of illness scores. Values are unadjusted for other covariates examined in the multivariable analysis. Shaded areas represent the $95 \%$ confidence interval around the smoothed regression line. CAT, Client Assessment of Treatment Scale.

attribution of symptoms. ${ }^{30}$ Furthermore, the construct of unawareness of illness varies in severity and over time and, although related to, is not interchangeable with symptom severity or treatment adherence. ${ }^{31}$ The potential importance of awareness of illness as a predictor variable for greater satisfaction with care is strengthened in this study because several additional potential predictor variables were included in the multivariable model, the relationship was present both at baseline during acute admission and at recovery on follow-up and patients who displayed improved awareness of illness over time also displayed greater satisfaction levels with their involuntary care when they considered it retrospectively.

The finding that a higher level of symptoms at baseline was associated with greater satisfaction with care at follow-up is likely because these individuals had a greater scope to improve their symptoms over time, as there was a statistical trend for a lower level of symptoms at baseline to be associated with greater satisfaction toward care at baseline, and greater reduction in symptoms over time was associated with change to higher level of satisfaction. This is consistent with a review of 18 mostly retrospective studies ${ }^{6}$ that reported that those individuals who had the greatest clinical gains in their admission had the most positive attitudes toward their involuntary admission and care retrospectively, and with a multicentre study by Katsakou et $a l,{ }^{9}$ who reported that greater improvement in symptoms over time was associated with higher levels of satisfaction, using the same satisfaction scale.

Individuals who were older had greater levels of satisfaction with the process and experience of involuntary admission at baseline and at follow-up, which is similar to some previous analyses ${ }^{11,32}$ but not all. ${ }^{29}$ A potential interpretation is that older individuals are more likely to have experienced other exposures to mental health services as out-patients or voluntary patients, and previous positive experiences may have softened their attitudes toward involuntary care. The association between a higher number of previous involuntary admissions and lower satisfaction with care at follow-up may be because individuals who had more involuntary admissions also had more severe or resistant mental illness and/or were more likely to default from community follow-up, reflecting a more negative relationship with the mental health services in general. In a 


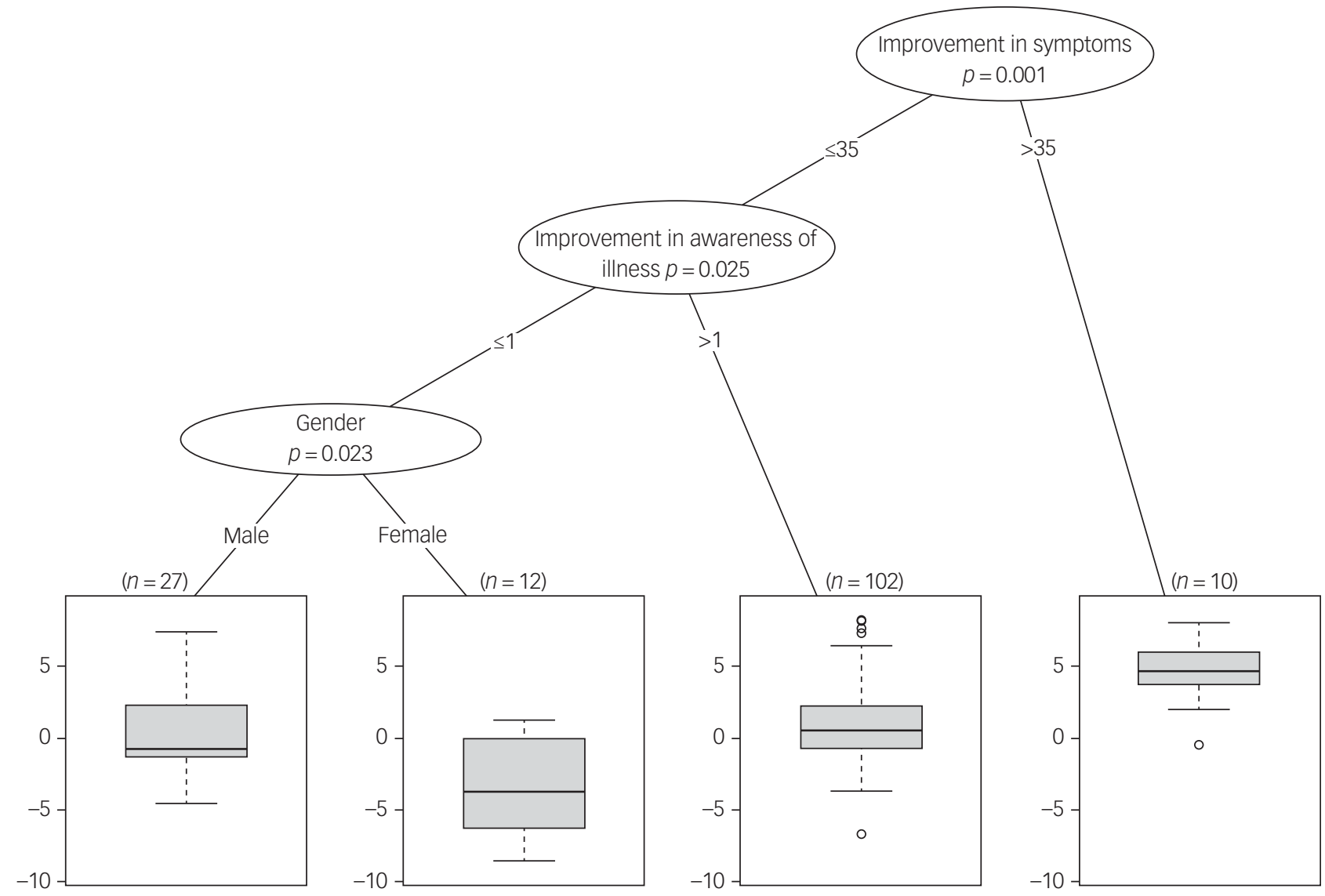

Fig. 2 Regression tree indicating optimal cut-off points for prediction of change in satisfaction with care over time. The scores on these scales were used to divide the participants who took part at both time points into groups based on how much their satisfaction with care changed over time (change in Client Assessment of Treatment Scale score, $y$-axis of each node). Improvement in symptoms refers to change in total Brief Psychiatric Rating Scale score from baseline to follow-up. Improvement in awareness of illness refers to change in total Schedule for the Assessment of Insight in Psychosis scores between baseline and follow-up.

longitudinal study by Setkowski et $a l,{ }^{33}$ a history of involuntary admissions was the only independent risk factor that predicted involuntary readmission.

Other factors more weakly associated with satisfaction with care were history of alcohol misuse, with greater satisfaction with care on initial assessment but not thereafter, and nonpsychotic diagnoses, with a change to greater satisfaction with care between baseline and follow-up. The regression tree analysis also identified a modulating effect of gender, consistent with some other reports of more negative views by women toward their care. ${ }^{7,32}$

We did not find any association between experience of objective coercive measures and satisfaction with involuntary admission. Smith et al found that individuals who had experienced seclusion were less satisfied, but there was no statistically significant association between the experience of restraint or forced intramuscular injection and attitudes toward involuntary admission and treatment. O'Donoghue et $a l^{8}$ did not find any association between the experience of physical coercion and whether or not the individual believed that their involuntary admission had been necessary at their 1 year follow-up. In contrast, a study by Iversen et $a l^{11}$ found that individuals' satisfaction with treatment decreased as the number of coercive events increased. In a study by Strauss et $a l^{28}$ aside from involuntary admission itself, the only coercive practice linked to lower satisfaction levels in in-patients was selfreported history of being refused a requested medication. McLaughlin et al reported, in a large multicentre study, that the experience of forced intramuscular medication was associated with a lower likelihood of patients retrospectively considering their involuntary admission as justifiable. ${ }^{12}$ Recruitment and follow-up rates are similar in our study to this multicentre study, and the differing findings in relation to coercive practices may be due to cultural or other methodological factors; for example, a lower proportion of patients in our study (30\%) were subject to forced intramuscular medication. In general, findings from this study were in keeping with the majority of previous studies, in that experience of objective coercive measures were not associated with poorer satisfaction among detained patients, but it is rather perceived coercion that is associated with negative attitudes toward involuntary admission and treatment. ${ }^{5,9,28}$ This suggests that measures taken to reduce the level of perceived coercion experienced by individuals in the process of being involuntarily admitted might increase patient satisfaction with their care, and treatment overall. Examples of positive practices that could be associated with higher satisfaction include enhanced therapeutic relationships, ${ }^{34}$ ensuring patients feel listened to ${ }^{2}$ and maximising opportunities for individuals to regain a sense of control. ${ }^{35}$ Optimising patient satisfaction with care during the difficult involuntary admission process is important not just for providing the highest quality and humane service to patients who require coercive care, but also because low satisfaction levels are associated with subsequent poor engagement with services and repeat involuntary admission. 27,33,36 Notably, a recent randomised controlled trial of individualised 
psychoeducation demonstrated reduced involuntary readmission rates in the intervention group, ${ }^{37}$ indicating beneficial effects of engagement and support for such high-risk individuals.

\section{Strengths and limitations}

A major strength of this study is that it was a large prospective study of a representative cohort from a defined geographical region with a relatively high follow-up rate. The in-patient units included reflected the differing types of in-patient care in Ireland. The distribution of the sample of participants in terms of age, gender and type of applicant was similar to the national figures reported by the Mental Health Commission, ${ }^{38}$ supporting the representative nature of the sample. A broad range of observer-rated structured assessments were included blind to the participants' attitudes ratings.

However, the study also had a number of limitations. A proportion of individuals refused to participate and it is possible that they had higher rates of dissatisfaction with the care received. Furthermore, $41 \%$ of individuals were lost to follow-up. Such individuals are difficult to retain in observational studies and this rate of loss to follow-up is within the range of other such studies. ${ }^{8,12,27,33}$ Individuals lost to follow-up were clinically similar at baseline to those who participated, suggesting that selection bias was not a major factor in the sample. Some data were not collected on all participants, in particular because those with lower satisfaction levels tended to leave out certain subscale items on the CAT, or because the scale was not applicable to the disorder (the SAI-E was designed for patients with psychotic illness). A small proportion of individuals (13\%) participated more than once and hence their data was not independent. This small cohort is unlikely to be driving the associations identified in the study, however, and these individuals may have had different experiences throughout their separate admissions. Participants were re-recruited at a time point when they were out-patients and had improved symptomatically, and this may have been at a point in their illness course when they had more favourable views toward their care.

This was an exploratory observational study and was not powered to test a specific hypothesis; three multivariable analysis were performed, but these were on different response variables, reflecting the longitudinal nature of the data collection and with pre-specified explanatory variables. Although the multivariable models explained $27-53 \%$ of the variance in the outcome measure, which is common for such analyses, a substantial amount of the variance in satisfaction with care remained unexplained, and there may be other relevant clinical factors that were not assessed. Although levels of symptoms and functioning were included as covariates, the analysis did not account for other indices of severity of the illness episode, such as total length of admission or time between termination of involuntary admission and reassessment. The wide variation in satisfaction levels reflects a heterogeneous group of respondents and more homogenous clinical subgroups may exist that were not identified by the current analysis.

Future work should include assessing the clinical determinants of satisfaction with care in non-detained individuals to determine what aspects of dissatisfaction may be specifically related to the experience of involuntary admission as distinct from other aspects of clinical care. Research into interventions to improve the experience of coercive care is sparse but feasible, with randomised controlled trials providing preliminary support for patient-centred care planning. ${ }^{39}$ The current study highlights the heterogenous nature of patient satisfaction with care during involuntary admission, and suggests that it may be fruitful to apply a stratified approach to future clinical trials by targeting for intervention those individuals likely to have persistently negative attitudes toward care, and therefore poor engagement with services. Hence, patients displaying low awareness of illness could be identified early in the course of their involuntary admission and targeted for recruitment into clinical trials to test the efficacy of specific interventions on their satisfaction with care and future engagement with community services. Potential interventions for such research include programmes to deliver individual psychoeducation, engage with peer support, optimise therapeutic relationships with clinicians and maximise opportunities for patient autonomy and decisionmaking.

In conclusion, although the majority of people admitted involuntarily retrospectively view their admission as justified, there is a wide variation in satisfaction with care, with many holding persistently low levels of satisfaction with their experience of involuntary admission. The strongest predictors of greater satisfaction with care and improvement in satisfaction over time were older age and clinical factors such as awareness of illness and improvements in awareness of illness and in symptoms. Such individuals likely to experience persistently low satisfaction with care on the basis of clinical measures assessed during involuntary admission could be identified for additional supportive interventions in an attempt to minimise the negative effect of involuntary admission and to enhance their engagement in supportive care thereafter.

Emma Bainbridge, MB, MRCPsych, MD, Honorary Clinical Fellow, College of Medicine, Nursing and Health Sciences, National University of Ireland Galway, Ireland; Brian Hallahan, MB MRCPsych MD, Senior Lecturer in Psychiatry, College of Medicine, Nursing and Health Sciences, National University of Ireland Galway, Ireland;

David McGuinness, MSc, PhD, Research Nurse, College of Medicine, Nursing and

Health Sciences, National University of Ireland Galway, Ireland; Patricia Gunning, PhD, Clinical Research Biostatistician, HRB Clinical Research Facility, National University of Ireland Galway, Ireland; John Newell, PhD, Professor of Biostatistics, HRB Clinical Research Facility, National University of Ireland Galway and School of Mathematics, Statistics and Applied Mathematics, National University of Ireland Galway, Ireland; Agnes Higgins, MSC, PhD (iD, Professor in Mental Health, School of Nursing and Midwifery, Trinity College Dublin, Ireland; Kathy Murphy, MSc PhD, Professor of Nursing, School of Nursing and Midwifery, National University of Ireland Galway, Ireland; Colm McDonald, MB, MRCPsych, PhD (D), Professor of Psychiatry, College of Medicine, Nursing and Health Sciences, National University of Ireland Galway and HRB Clinical Research Facility, National University of Ireland Galway, Ireland

Correspondence: Colm McDonald, Clinical Science Institute, National University of Ireland, Galway, H91 YR71, Ireland. Email: colm.mcdonald@nuigalway.ie

First received 20 Dec 2017, final revision 12 Sep 2018, accepted 7 Oct 2018

\section{Funding}

This study was funded by the Mental Health Commission: Research Programme Grants in Mental Health Research awarded to Colm McDonald.

\section{Acknowledgements}

We acknowledge the contributions of all patients who gave their time to participate in this study and clinical colleagues who assisted with recruitment. We are most grateful to Dr Liz Brosnan, Dr Mary Keys and Dr Heike Felzmann for contributing to the project steering group, which guided the research programme underpinning this study.

\section{References}

1 Naber D, Kircher T, Hessel K. Schizophrenic patients' retrospective attitudes regarding involuntary psychopharmacological treatment and restraint. Eur Psychiatry 1996; 11(1): 7-11.

2 Katsakou C, Priebe S. Patient's experiences of involuntary hospital admission and treatment: a review of qualitative studies. Epidemiol Psichiatr Soc 2007; 16(2): 172-8

3 Thornicroft G, Farrelly S, Birchwood M, Marshall M, Szmukler G, Waheed W, et al. CRIMSON [CRisis plan IMpact: subjective and Objective coercion and eNgagement] protocol: a randomised controlled trial of joint crisis plans to reduce compulsory treatment of people with psychosis. Trials 2010; 11: 102. 
4 Wyder M, Bland R, Crompton D. The importance of safety, agency and control during involuntary mental health admissions. J Ment Health 2016; 25(4): 338-42.

5 Smith D, Roche E, O'Loughlin K, Brennan D, Madigan K, Lyne J, et al. Satisfaction with services following voluntary and involuntary admission. $J$ Ment Health 2014; 23(1): 38-45.

6 Katsakou C, Priebe S. Outcomes of involuntary hospital admission-a review. Acta Psychiatr Scand 2006; 114(4): 232-41.

7 Priebe S, Katsakou C, Glockner M, Dembinskas A, Fiorillo A, Karastergiou A et al. Patients' views of involuntary hospital admission after 1 and 3 months: prospective study in 11 European countries. Br J Psychiatry 2010; 196(3): 179-85.

8 O'Donoghue B, Lyne J, Hill M, O'Rourke L, Daly S, Larkin C, et al. Perceptions of involuntary admission and risk of subsequent readmission at one-year followup: the influence of insight and recovery style. J Ment Health 2011; 20(3): 249-59.

9 Katsakou C, Bowers L, Amos T, Morriss R, Rose D, Wykes T, et al. Coercion and treatment satisfaction among involuntary patients. Psychiatr Serv 2010; 61(3): 286-92.

10 O'Donoghue B, Lyne J, Hill M, Larkin C, Feeney L, O'Callaghan E. Physical coercion, perceived pressures and procedural justice in the involuntary admission and future engagement with mental health services. Eur Psychiatry 2011; 26(4): 208-14.

11 Iversen KI, Høyer G, Sexton HC. Coercion and patient satisfaction on psychiatric acute wards. Int J Law Psychiatry 2007; 30(6): 504-11.

12 McLaughlin P, Giacco D, Priebe S. Use of coercive measures during involuntary psychiatric admission and treatment outcomes: data from a prospective study across 10 European Countries. PLOS ONE 2016; 11(12): e0168720.

13 Giacco D, Mavromara L, Gamblen J, Conneely M, Priebe S. Shared decisionmaking with involuntary hospital patients: a qualitative study of barriers and facilitators. BJPsych Open 2018; 4(3): 113-8.

14 Dressing $\mathrm{H}$, Salize HJ. Compulsory admission of mentally ill patients in European Union Member States. Soc Psychiatry Psychiatr Epidemiol 2004; 39(10): 797-803.

15 Overall JE, Gorham DR. The Brief Psychiatric Rating Scale. Psychol Rep 1962; 10: $799-12$

16 Hall RC. Global Assessment of Functioning. A modified scale. Psychosomatics 1995; 36(3): 267-75.

17 Kemp RA, David AS. Insight and adherence to treatment in psychotic disorders. Br J Hosp Med 1995; 54(5): 222-7.

18 Hogan TP, Awad AG, Eastwood R. A self-report scale predictive of drug compliance in schizophrenics: reliability and discriminative validity. Psychol Med 1983; 13(1): 177-83.

19 Priebe S, Gruyters T. Patients' assessment of treatment predicting outcome. Schizophr Bull 1995; 21(1): 87-94.

20 Gardner W, Hoge SK, Bennett N, Roth LH, Lidz CW, Monahan J, et al. Two scales for measuring patients' perceptions for coercion during mental hospital admission. Behav Sci Law 1993; 11(3): 307-21.

21 Iverson KI, Hoyer G, Sexton H, Gronli OK. Perceived coercion among patients admitted to acute wards in Norway. Nord J Psychiatry 2002; 56: 433-9.
22 American Psychiatric Association. Diagnostic and Statistical Manual of Mental Disorders. 4th ed. APA, 2000

23 Heinrichs DW, Hanlon TE, Carpenter WT Jr. The Quality of Life Scale: an instrument for rating the schizophrenic deficit syndrome. Schizophr Bull 1984; 10(3): 388-98.

24 Hothorn T, Hornik K, Zeileis A. Unbiased recursive partitioning: a conditional inference framework. J Comput Graphical Stat 2006; 15(3): 651-74.

25 R Development Core Team. R: A Language and Environment for Statistical Computing. R Foundation for Statistical Computing, 2013

26 Kilbourne AM, McCarthy JF, Post EP, Welsh D, Pincus HA, Bauer MS, et al. Access to and satisfaction with care comparing patients with and without serious mental illness. Int J Psychiatry Med 2006; 36(4): 383-99.

27 Priebe S, Katsakou C, Amos T, Leese M, Morriss R, Rose D, et al. Patients' views and readmissions 1 year after involuntary hospitalisation. Br J Psychiatry 2009; 194(1): 49-54.

28 Strauss JL, Zervakis JB, Stechuchak KM, Olsen MK, Swanson J, Swartz MS, et al Adverse impact of coercive treatments on psychiatric inpatients' satisfaction with care. Community Ment Health J 2013; 49(4): 457-65.

29 O'Donoghue B, Lyne J, Hill M, Larkin C, Feeney L, O'Callaghan E. Involuntary admission from the patients' perspective. Soc Psychiatry Psychiatr Epidemiol 2010; 45(6): 631-8

30 David AS. 'To see oursels as others see us". Aubrey Lewis's insight. $\mathrm{Br}$ J Psychiatry 1999; 175: 210-16.

31 Mintz AR, Dobson KS, Romney DM. Insight in schizophrenia: a meta-analysis Schizophr Res 2003; 61(1): 75-88.

32 Savill M, Jankovic J, Katsakou C, Kallert T, Priebe S. Symptom levels and initial appraisal of hospital treatment in patients with schizophrenia. Psychiatry Res 2012; 199(2): 79-83.

33 Setkowski K, van der Post LF, Peen J, Dekker JJ. Changing patient perspectives after compulsory admission and the risk of re-admission during 5 years of follow-up: The Amsterdam Study of Acute Psychiatry IX. Int J Soc Psychiatry. 2016; 62(6): 578-88.

34 Roche E, Madigan K, Lyne JP, Feeney L, O'Donoghue B. The therapeutic relationship after psychiatric admission. J Nerv Ment Dis 2014; 202(3): 186-92.

35 McGuinness D, Higgins A, Bainbridge E, Brosnan L, Keys M, Felzmann H, et al. Preserving control - a qualitative study of individuals' experiences of involuntary admission. this issue.

36 van der Post LF, Peen J, Visch I, Mulder CL, Beekman AT, Dekker JJ. Patient perspectives and the risk of compulsory admission: the Amsterdam Study of Acute Psychiatry V. Int J Soc Psychiatry. 2014; 60(2): 125-33.

37 Lay B, Kawohl W, Rossler W. Outcomes of a psycho-education and monitoring programme to prevent compulsory admission to psychiatric inpatient care: a randomised controlled trial. Psychol Med 2018; 48(5): 849-60.

38 Mental Health Commission. Annual Report 2014 Including Report of the Inspector of Mental Health Services. Mental Health Commission, 2014.

39 Giacco D, Conneely M, Masoud T, Burn E, Priebe S. Interventions for involuntary psychiatric inpatients: a systematic review. Eur Psychiatry 2018; 54: $41-50$. 